\title{
Concentration and Density Changes at an Electrode Surface and the Principle of Unchanging Total Concentration*
}

\author{
Stephen W. Feldberg and Ernie R. Lewis \\ feldberg@bnl.gov; elewis@bnl.gov \\ Brookhaven National Laboratory Upton, NY 11973
}

Accepted for publication in Journal of the Electrochemical Society

\begin{abstract}
.
The principle of unchanging total concentration as described by Oldham and Feldberg ${ }^{1}$ is invoked to analyze systems comprising a redox pair $\left(X_{1}^{z_{1}}\right.$ and $\left.X_{2}^{z_{2}}\right)$ plus one or more nonelectroactive ions $\left(\mathrm{X}_{3}^{z_{3}}, \mathrm{X}_{4}^{z_{4}} \ldots \mathrm{X}_{\mathrm{jmax}}^{z_{\mathrm{j} \max }}\right)$. The principle states that:

$$
S_{\mathrm{P}}=\sum_{\mathrm{j}=1}^{\mathrm{j}_{\max }}\left[\mathrm{X}_{\mathrm{j}}^{z_{\mathrm{j}}}\right]=\sum_{\mathrm{j}=1}^{\mathrm{j}_{\max }} c_{\mathrm{j}}=\sum_{\mathrm{j}=1}^{\mathrm{j}_{\max }} c_{\mathrm{j}}^{\text {bulk }}
$$
\end{abstract}

In this particular application the "principle" allows quantification of the electrochemically induced concentration changes at the electrode surface given the requirement that all difffusion coefficients are identical, the bulk concentrations, the surface boundary condition $\left(c_{2}^{x=0} / c_{1}^{x=0}\right)$ the constraint of electroneutrality, and a Boltzmann-like relationship correlating surface $c_{\mathrm{j} \geq 3}^{x=0}$ and bulk $c_{\mathrm{j} \geq 3}^{\text {bulk }}$ concentrations for species $\mathrm{j} \geq 3$. The surface concentrations coupled with known values of the partial molar volumes and molecular masses, can provide an estimate of the density gradients within the depletion region at electrode surface; those gradients in turn can induce convection.

\section{Introduction.}

The operative mathematical constraint of the principle of unchanging total concentration (henceforth to be referred to as "the principle" or as "the constraint of eqn. 1" or simply as $S_{\mathrm{P}}$ ) was discussed by Oldham and Feldberg:

*This work is dedicated to Prof. Allen J. Bard - a revered colleague and a revered friend. 


$$
S_{\mathrm{P}}=\sum_{\mathrm{j}=1}^{\mathrm{j}_{\max }}\left[\mathrm{X}_{\mathrm{j}}^{z_{\mathrm{j}}}\right]=\sum_{\mathrm{j}=1}^{\mathrm{j}_{\max }} c_{\mathrm{j}}=\sum_{\mathrm{j}=1}^{\mathrm{j}_{\max }} c_{\mathrm{j}}^{\text {bulk }}
$$

where $c_{\mathrm{j}}\left(\right.$ moles $\left.\mathrm{cm}^{-3}\right)$ is the concentration of the $\mathrm{j}^{\mathrm{t}}$ species at any point $(x, y, z)$ in the solution. Eqn. 1 holds as long as:

- diffusion coefficients of all species are identical.

- electroneutrality obtains throughout the solution (deviations from electroneutrality in the vicinity of the double layer are ignored):

$$
\sum_{j=1}^{\mathrm{j}_{\max }} c_{\mathrm{j}} z_{\mathrm{j}}=0
$$

- transport is governed by the Nernst-Planck expression (modified to account for convection if needed):

$\vec{f}_{\mathrm{j}}=-D \nabla c_{\mathrm{j}}-\frac{F}{R T} D c_{\mathrm{j}} z_{\mathrm{j}} \nabla \Phi+\vec{v} c_{\mathrm{j}}$

Where $\Phi$ potential at $x, y$ and $z, D\left(\mathrm{~cm}^{2} / \mathrm{s}\right)$ is the common diffusion coefficient, $v(\mathrm{~cm} / \mathrm{s})$ is the solution velocity, $F, R$ and $T$ have their usual significance; the operator $\nabla$ is defined by:

$$
\nabla=\frac{\partial}{\partial x}+\frac{\partial}{\partial y}+\frac{\partial}{\partial z}
$$

- solute numbers remain constant, i.e., no homogeneous reactions of the form $A \Leftrightarrow n B$; no heterogeneous reactions of the form $\mathrm{nB} \pm \mathrm{e} \Leftrightarrow \mathrm{A}$ (where $\mathrm{n} \geq 2$ ), no ion pairing, no desorption-adsorption processes.

When these criteria obtain, the validity of eqn. 1 is independent of the electrode size and shape and of the electrochemical protocol.

The experiment of interest involves a system with an initially present redox moiety $X_{1}^{z_{1}}$ at bulk concentration $c_{1}^{\text {bulk }}$; the concentration of the complementary $\mathrm{X}_{2}^{z_{2}}$ redox species will usually be zero but need not be. The notational protocol reserves indices 1 and 2 for the redox pair and indices $\geq 3$ for any number of additional electroinactive ionic species: $X_{j \geq 3}^{z_{j 23}}$. Bulk concentrations 
$c_{\mathrm{j} \geq 3}^{\text {bulk }}$ are set to meet the condition of electroneutrality (eqn. 2 ). In principle, those species $\mathrm{j} \geq 3$ can be neutral but that case is uninteresting and would produce $c_{\mathrm{j}}^{x=0}=c_{\mathrm{j}}^{\text {bulk }}$. This will become clearer in later discussion.

The initial boundary condition is consistent with the ratio $c_{2}^{x=0} / c_{1}^{x=0}=c_{2}^{\text {bulk }} / c_{1}^{\text {bulk }}$. The electrochemical perturbation of the system is that required to reset the ratio $c_{2}^{x=0} / c_{1}^{x=0} \Rightarrow \theta_{\mathrm{D}}$ defined by.

$$
\theta_{\mathrm{D}}=\frac{\left[\mathrm{X}_{2}^{z_{2}}\right]_{x=0}}{\left[\mathrm{X}_{1}^{z_{1}}\right]_{x=0}}=\frac{c_{2}^{x=0}}{c_{1}^{x=0}}
$$

Alternatively one can also define $\alpha_{\mathrm{D}}$ (the fraction of conversion of $\mathrm{X}_{1}^{z_{1}}$ to $\mathrm{X}_{2}^{z_{2}}$ at $x=0$ ):

$$
\alpha_{\mathrm{D}}=\frac{\theta_{\mathrm{D}}}{1+\theta_{\mathrm{D}}} \text { or } \theta_{\mathrm{D}}=\frac{\alpha_{\mathrm{D}}}{1-\alpha_{\mathrm{D}}}
$$

In principle, the value of $\theta_{\mathrm{D}}$ (or) could be stepped to any desired new value by changing the electrode potential - easily accomplished if the redox couple is reversible and if there is no significant uncompensated resistance $\left(R_{\mathrm{u}}\right)$ - unlikely with low supporting electrolyte. However, $R_{\mathrm{u}}$ can be minimized by clever cell design. Diminishing $R_{\mathrm{u}}$ electronically by positive feedback. ${ }^{2}$ is not so easily accomplished since $R_{\mathrm{u}}$ is a function of solution composition which may be changing as a function of time. If the only interest is the limiting case where $\theta_{\mathrm{D}} \Rightarrow \infty$ that can be accomplished rather straightforwardly by setting $E_{\text {applied }}-E^{0}$ sufficiently positive (for oxidation of $X_{1}^{z_{1}}$ to $X_{2}^{z_{2}}$ ) or sufficiently negative (for reduction of $X_{1}^{z_{1}}$ to $X_{2}^{z_{2}}$ ).

The objective of the present work is twofold:

1. Use the principle of unchanging total concentration to deduce all the surface concentrations $c_{\mathrm{j}}^{x=0}\left(\right.$ moles $\left.\mathrm{cm}^{-3}\right)$ given the value of $\theta_{\mathrm{D}}$, all the $c_{\mathrm{j}}^{\text {bulk }}\left(\right.$ moles $\left.\mathrm{cm}^{-3}\right)$ values and their associated charges $z_{\mathrm{j}}$.

2. Use the $c_{\mathrm{j}}^{x=0}$ and $c_{\mathrm{j}}^{\text {bulk }}$ values to estimate the difference between the density of the solution at $x=0$ and the density of the bulk solution. This may help to sharpen the 
distinctions between convection produced by electrochemically induced density changes significant and the "spontaneous" or natural convection ${ }^{3}$

\section{Theory:}

In the present analysis the principle of unchanging total concentration is invoked to deduce all the surface concentrations $c_{\mathrm{j}}^{x=0}$ following a change in the electrode potential to effect $\theta_{\mathrm{D}}$ at the electrode surface (see eqn. 4). Heretofore, only the solution for the 3 -species problem has been discussed, see e.g. Oldham and Feldberg ${ }^{1}$. The present analysis reveals a straightforward (albeit iterative) solution to compute $c_{\mathrm{j} \geq 2}^{x=0}$ when $\mathrm{j}_{\max } \geq 3$. The 3 -species problem is a subset of that more complete treatment. The earlier study of the principle evolved from our interest at the time in systems involving little or no added supporting electrolyte. Numerous works have focused on fluxes $D\left(\mathrm{~d} c_{\mathrm{j}} / \mathrm{d} x\right)_{x=0}$ as a function of these same variables ${ }^{4-8}$; however, it is the values of $c_{\mathrm{j}}^{x=0}$ (and not the fluxes) that are of primary interest in the present work because of their relevance to the density of the solution in the depletion region adjacent to the electrode surface.

Consequently, I do not attempt to deduce the fluxes (and currents) - a complex analysis; the results will depend upon the size and shape of the electrode, time (if the system is not at steadystate) and upon the electrochemical protocol - arguably unnecessary for the present purposes.

\section{The 3-species problem.}

It is instructive to review first the solution for the 3 -species problem which can be solved explicitly. ${ }^{1} S_{\mathrm{P}}$ is directly evaluated from eqn. 1 :

$$
S_{\mathrm{P}}=\sum_{j=1}^{3} c_{\mathrm{j}}^{\text {bulk }}
$$

It is also the case that:

$$
S_{\mathrm{P}}=\sum_{j=1}^{3} c_{\mathrm{j}}^{\mathrm{bulk}}=c_{1}^{x=0}+c_{2}^{x=0}+c_{3}^{x=0}=\frac{c_{2}^{x=0}}{\theta_{\mathrm{D}}}+c_{2}^{x=0}+c_{3}^{x=0}=\left(\frac{1}{\theta_{\mathrm{D}}}+1\right) c_{2}^{x=0}+c_{3}^{x=0}
$$

With rearrangement this becomes: 
$c_{3}^{x=0}=S_{\mathrm{P}}-\left(1+\frac{1}{\theta_{\mathrm{D}}}\right) c_{2}^{x=0}$

Invoking the electroneutrality constraint (eqn. 2) and eqn. 7 leads to:

$0=c_{1}^{x=0} z_{1}+c_{2}^{x=0} z_{2}+c_{3}^{x=0} z_{3}=\left(\frac{z_{1}}{\theta_{\mathrm{D}}}+z_{2}\right) c_{2}^{x=0}+c_{3}^{x=0} z_{3}$

Then:

$c_{3}^{x=0}=-\frac{\left(\frac{z_{1}}{\theta_{\mathrm{D}}}+z_{2}\right)}{z_{3}} c_{2}^{x=0}$

Equating eqns. 8 and 11 gives

$S_{\mathrm{P}}=\left(1+\theta_{\mathrm{D}}\right) c_{2}^{x=0}+c_{2}^{x=0} \frac{\frac{z_{1}}{\theta_{\mathrm{D}}}+z_{2}}{z_{3}}=c_{2}^{x=0}\left(\left(1+\frac{1}{\theta_{\mathrm{D}}}\right)+\frac{\frac{z_{1}}{\theta_{\mathrm{D}}}+z_{2}}{z_{3}}\right)$

Then:

$$
\begin{gathered}
c_{2}^{x=0}=\frac{S_{\mathrm{P}}}{\left(\left(1+\frac{1}{\theta_{\mathrm{D}}}\right)-\frac{\left(\frac{z_{1}}{\theta_{\mathrm{D}}}+z_{2}\right)}{z_{3}}\right)}=\frac{S_{\mathrm{P}} z_{3}}{z_{3}\left(1+\frac{1}{\theta_{\mathrm{D}}}\right)-\left(\frac{z_{1}}{\theta_{\mathrm{D}}}+z_{2}\right)} \\
c_{1}^{x=0}=\frac{c_{2}^{x=0}}{\theta_{\mathrm{D}}}=\frac{\theta_{\mathrm{D}}\left(z_{3}\left(1+\frac{1}{\theta_{\mathrm{D}}}\right)-\left(\frac{z_{1}}{\theta_{\mathrm{D}}}+z_{2}\right)\right)}{S_{\mathrm{P}} z_{3}} \\
c_{3}^{x=0}=S_{\mathrm{P}}-\left(c_{1}^{x=0}+c_{2}^{x=0}\right)=-\frac{S_{\mathrm{P}}\left(z_{1}+\theta_{\mathrm{D}} z_{2}\right)}{z_{3}\left(1+\theta_{\mathrm{D}}\right)-\left(z_{1}+\theta_{\mathrm{D}} z_{2}\right)}
\end{gathered}
$$


The results for two examples of 3-species systems are summarized in Table 1. For example 1A the two species are a singly positively charged redox moiety and its corresponding negatively charged counter ion. For this example, species 2 has zero charge so the redox process is a 1electron reduction. The species distributions are shown for $\alpha_{\mathrm{D}}=0.25,0.5,0.75$ and 0.999999 . The possibly surprising result is that the final concentration of $c_{2}^{x=0}=2 c_{1}^{\text {bulk }}$, a result that has been theoretically explored and discussed ${ }^{1}$ along with several other 3 -species variations. ${ }^{1}$ For the example shown in Table 1B the initial species are the same as for 1A. However, the redox product is now doubly positively charged so the redox process was a 1-electron oxidation.

The general solution for $\mathrm{j}_{\max } \geq 3$.

The 3-species problem involved evaluation of three unknowns $\left(c_{1}^{x=0}, c_{2}^{x=0}\right.$ and $\left.c_{3}^{x=0}\right)$ from three equations (eqns. 1, 2 and 5). For each added species there will be an additional unknown whose evaluation requires an additional equation. When the surface fluxes are zero, as they must be at all times for species $\mathrm{j} \geq 3$, the Nernst-Planck equation (eqn. 3) leads to the Boltzmann relationship for those species:

$c_{\mathrm{j} \geq 3}^{x=0}=c_{\mathrm{j} \geq 3}^{\text {bulk }} \exp \left(-\frac{F}{R T} \Phi z_{\mathrm{j} \geq 3}\right)=c_{\mathrm{j} \geq 3}^{\text {bulk }} \exp (-\gamma)$

where:

$\gamma=\frac{F}{R T} \Phi$

Eqn. $\quad 16$ is reminiscent of the expression used to derive the Gouy-Chapman equation, ${ }^{2}$ but without the constraint of electroneutrality. Note that when $z_{\mathrm{j} \geq 3}=0 \quad c_{\mathrm{j} \geq 3}^{x=0}=c_{\mathrm{j} \geq 3}^{\mathrm{bulk}}$.

The constraints of eqns. 1 and 2 are now:

$S_{\mathrm{P}}=\sum_{\mathrm{j}=1}^{\mathrm{jmax}} c_{\mathrm{j}}^{\mathrm{bulk}}=\sum_{\mathrm{j}=1}^{\mathrm{jmax}} c_{\mathrm{j}}^{x=0}$

and 
$\sum_{\mathrm{j}=1}^{\mathrm{jmax}} c_{\mathrm{j}}^{\text {bulk }} z_{\mathrm{j}}=\sum_{\mathrm{j}=1}^{\mathrm{jmax}} c_{\mathrm{j}}^{x=0} z_{\mathrm{j}}=0$

Following the approach described in the 3-species model and introducing the boundary condition (eqn. 5):

$S_{\mathrm{P}}-\sum_{\mathrm{j}=3}^{\operatorname{jmax}} c_{\mathrm{j}}^{x=0}=c_{1}^{x=0}+c_{2}^{x=0}=\frac{c_{2}^{x=0}}{\theta_{\mathrm{D}}}+c_{2}^{x=0}=c_{2}^{x=0}\left(\frac{1}{\theta_{\mathrm{D}}}+1\right)$

Rearranging and introducing the Boltzmann relationship (eqn.

16):

$c_{2}^{x=0}=\frac{S_{\mathrm{P}}-\sum_{\mathrm{j}=3}^{\mathrm{j} \max } c_{\mathrm{j}}^{x=0}}{\left(\frac{1}{\theta_{\mathrm{D}}}+1\right)}=\frac{S_{\mathrm{P}}-\sum_{\mathrm{j}=3}^{\mathrm{jmax}} c_{\mathrm{j} \geq 3}^{\text {bulk }} \exp \left(-\gamma z_{\mathrm{j} \geq 3}\right)}{\left(\frac{1}{\theta_{\mathrm{D}}}+1\right)}$

Invoking the charge neutrality constraint, eqn. 4, gives:

$$
0=c_{1}^{x=0} z_{1}+c_{2}^{x=0} z_{2}+\sum_{\mathrm{j}=3}^{\text {jmax }} z_{\mathrm{j}} c_{\mathrm{j}}^{\text {bulk }} \exp \left[-\gamma z_{\mathrm{j}}\right]=c_{2}^{x=0}\left(\frac{z_{1}}{\theta_{\mathrm{D}}}+z_{2}\right)+\sum_{\mathrm{j}=3}^{\mathrm{jmax}} z_{\mathrm{j}} c_{\mathrm{j}}^{\text {bulk }} \exp \left[-\gamma z_{\mathrm{j}}\right]
$$

Then

$$
c_{2}^{x=0}=-\frac{\sum_{\mathrm{j}=3}^{\mathrm{jmax}} z_{\mathrm{j}} c_{\mathrm{j}}^{\text {bulk }} \exp \left[-\gamma z_{\mathrm{j}}\right]}{\frac{z_{1}}{\theta_{\mathrm{D}}}+z_{2}}
$$

Combining eqns. 21 and 23 gives

$$
0=\frac{S_{\mathrm{P}}-\sum_{\mathrm{j}=3}^{\mathrm{j} \text { max }} c_{\mathrm{j}}^{\text {bulk }} \exp \left[-\gamma z_{\mathrm{j}}\right]}{\frac{1}{\theta_{\mathrm{D}}}+1}+\frac{\sum_{\mathrm{j}=3}^{\mathrm{jmax}} z_{\mathrm{j}} c_{\mathrm{j}}^{\text {bulk }} \exp \left[-\gamma z_{\mathrm{j}}\right]}{\frac{z_{1}}{\theta_{\mathrm{D}}}+z_{2}}
$$


or

$$
0=\left(\frac{z_{1}}{\theta_{\mathrm{D}}}+z_{2}\right)\left(S_{\mathrm{P}}-\sum_{\mathrm{j}=3}^{\mathrm{j}_{\max }} c_{\mathrm{j}}^{\text {bulk }} \exp \left[-\gamma z_{\mathrm{j}}\right]\right)+\left(\frac{1}{\theta_{\mathrm{D}}}+1\right) \sum_{\mathrm{j}=3}^{\mathrm{jmax}} z_{\mathrm{j}} c_{\mathrm{j}}^{\text {bulk }} \exp \left[-\gamma z_{\mathrm{j}}\right]
$$

When $\mathrm{j}_{\max }=3 \gamma$ can be solved for directly. That is easily seen by noting that for $\mathrm{j}_{\max }=3$ eqn. 24 can be written as:

$$
0=\left(\frac{z_{1}}{\theta_{\mathrm{D}}}+z_{2}\right)\left(S_{\mathrm{P}}-c_{3}^{\text {bulk }} \exp \left[-\gamma z_{3}\right]\right)+\left(\frac{1}{\theta_{\mathrm{D}}}+1\right) z_{3} c_{3}^{\text {bulk }} \exp \left[-\gamma z_{3}\right]
$$

Replacing $c_{3}^{\text {bulk }} \exp \left[-\gamma z_{3}\right]$ by $c_{3}^{x=0}($ see eqn. $\quad 16)$ and rearranging gives:

$$
c_{3}^{x=0}=-\frac{-\left(\frac{z_{1}}{\theta_{\mathrm{D}}}+z_{2}\right) S_{\mathrm{P}}}{z_{3}\left(\frac{1}{\theta_{\mathrm{D}}}+1\right)-\left(\frac{z_{1}}{\theta_{\mathrm{D}}}+z_{2}\right)}=-\frac{\left(z_{1}+z_{2} \theta_{\mathrm{D}}\right) S_{\mathrm{P}}}{z_{3}\left(1+\theta_{\mathrm{D}}\right)-\left(z_{1}+z_{2} \theta_{\mathrm{D}}\right)}
$$

Eqn. 27 is identical to eqn. 15 directly deduced for the 3 -species system. When $\mathrm{j}>3$ solving for the value of $\gamma$ (eqn. 26) must be solved iteratively: the bisection approach is relatively easy to implement (e.g., compared to Newton-Raphson method) and is adequate for the present purposes. Some sample results are shown in Table 2 for 5 -species and 4-species systems.

A 5-species system allows straightforward examination of what happens when excess electrolyte is present in the system. Initially present in the system will be a redox moiety and it's counter-ion plus a much higher concentration of an additional pair of ions which constitute the supporting electrolyte. The result is that sum of the normalized concentrations of the redox species at $x=0$ is just what would be expected, i.e.: $c_{1}^{x=0} / c_{1}^{\text {bulk }}+c_{2}^{x=0} / c_{1}^{\text {bulk }}=1$. (see Table 2). This is definitively not the case when there is no added supporting electrolyte (see Table 1). Also shown in Table 2 are some values of the surface concentrations computed by direct explicit finite-difference simulation. ${ }^{7}$. We believe that this confirms that the Boltzmann expression, eqn. 16, is producing the correct and unique result. 
Computing the electrochemically induced density change $\Delta \rho$ at $x=0$.

In the previous segment I showed that the surface concentrations, $c_{\mathrm{j}}^{x=0}$, can be deduced given identical diffusion coefficients), set values of $c_{\mathrm{j}}^{\text {bulk }}$ associated with a particular mechanistic scheme and the value of $\theta_{\mathrm{D}}$ (or $\alpha_{\mathrm{D}}$ ). It is then possible to estimate the difference between the density of the solution at $x=0$ following the electrochemical perturbation and the density of the bulk solution if the single-ion or neutral partial molar volumes, $\beta_{\mathrm{j}}$, are known. To further simplify this analysis I assume that the partial molar density (PMD) and apparent molar density (AMD) are the same. Implicit in that assumption is the added assumption that the solute species behave independently and are additive. The density of the bulk solution will be:

$$
\rho^{\text {bulk }}=\frac{\rho^{\mathrm{S}}+\sum_{\mathrm{j}=1}^{\text {jmax }} M_{\mathrm{j}} c_{\mathrm{j}}^{\text {bulk }}}{1+\sum_{\mathrm{j}=1}^{\text {imax }} \beta_{\mathrm{j}} c_{\mathrm{j}}^{\text {bulk }}}
$$

where $\rho^{\mathrm{S}}\left(\mathrm{g} \mathrm{cm}^{-3}\right)$ is the density of the solvent and $M_{\mathrm{j}}\left(\mathrm{g} \mathrm{mole}^{-1}\right)$ is the molecular mass of species $\mathrm{j}$. The analogous equation can be written for the density at the surface:

$$
\rho^{x=0}=\frac{\rho^{\mathrm{S}}+\sum_{\mathrm{j}=1}^{\mathrm{jmax}} M_{\mathrm{j}} c_{\mathrm{j}}^{x=0}}{1+\sum_{\mathrm{j}=1}^{\text {jmax }} \beta_{\mathrm{j}} c_{\mathrm{j}}^{x=0}}
$$

The difference between the surface and bulk densities will be $\Delta \rho$ :

$$
\Delta \rho=\rho^{x=0}-\rho^{\text {bulk }}=\frac{\rho^{\mathrm{S}}+\sum_{\mathrm{j}=1}^{\mathrm{jmax}} M_{\mathrm{j}} c_{\mathrm{j}}^{x=0}}{1+\sum_{\mathrm{j}=1}^{\mathrm{max}} \beta_{\mathrm{j}} c_{\mathrm{j}}^{x=0}}-\frac{\rho^{\mathrm{S}}+\sum_{\mathrm{j}=1}^{\mathrm{jmax}} c_{\mathrm{j}}^{\text {bulk }}}{1+\sum_{\mathrm{j}=1}^{\mathrm{jmax}} \beta_{\mathrm{j}} c_{\mathrm{j}}^{\text {bulk }}}
$$

or 


$$
\frac{\Delta \rho}{\rho^{\mathrm{S}}}=\frac{1+\sum_{\mathrm{j}=1}^{\mathrm{j} \max } M_{\mathrm{j}} c_{\mathrm{j}}^{x=0} / \rho^{\mathrm{S}}}{1+\sum_{\mathrm{j}=1}^{\mathrm{j} \max } \beta_{\mathrm{j}} c_{\mathrm{j}}^{x=0}}-\frac{1+\sum_{\mathrm{j}=1}^{\mathrm{j} \max } c_{\mathrm{j}}^{\text {bulk }} / \rho^{\mathrm{S}}}{1+\sum_{\mathrm{j}=1}^{\text {max }} \beta_{\mathrm{j}} c_{\mathrm{j}}^{\text {bulk }}}
$$

For the most part the literature values of $\beta_{\mathrm{j}}$ are less than $\approx 10^{3} \mathrm{~cm}^{3} /$ mole (see, e.g. ${ }^{9-12}$ ), values of $M_{\mathrm{j}}$ are less than $\approx 10^{3} \mathrm{~g} /$ mole , assigned values of $c_{\mathrm{j}}^{\text {bulk }}$ and computed values of $c_{\mathrm{j}}^{x=0}$ are less than $\approx 10^{-5}$ moles $/ \mathrm{cm}^{3}(=0.01 \underline{\mathrm{M}})$. Thus the following set of conditions will generally be fulfilled:

$$
\begin{aligned}
& \frac{\sum_{\mathrm{j}=1}^{\mathrm{jmax}} M_{\mathrm{j}} c_{\mathrm{j}}^{x=0}}{\rho^{\mathrm{S}}} \leq 1 \\
& \left|\sum_{\mathrm{j}=1}^{\mid \operatorname{mmax}} \beta_{\mathrm{j}} c_{\mathrm{j}}^{x=0}\right| \leq 1 \\
& \frac{\sum_{\mathrm{j}=1}^{\mathrm{jmax}} M_{\mathrm{j}} c_{\mathrm{j}}^{\text {bulk }}}{\rho^{\mathrm{S}}} \leq 1 \\
& \left|\sum_{\mathrm{j}=1}^{\max } \beta_{\mathrm{j}} c_{\mathrm{j}}^{\text {bulk }}\right| \leq 1
\end{aligned}
$$

When these conditions are met (and they generally will be), eqn. 31 can be simplified to give:

$$
\Delta \rho / \rho^{\mathrm{s}} \cong\left(\sum_{\mathrm{j}=1}^{\mathrm{j} \max } \beta_{\mathrm{j}} c_{\mathrm{j}}^{\text {bulk }}+\sum_{\mathrm{j}=1}^{\mathrm{j} \max } M_{\mathrm{j}} \mathrm{c}_{\mathrm{j}}^{x=0} / \rho^{\mathrm{s}}\right)-\left(\sum_{\mathrm{j}=1}^{\mathrm{j} \max } \beta_{\mathrm{j}} c_{\mathrm{j}}^{x=0}+\sum_{\mathrm{j}=1}^{\mathrm{j} \max } M_{\mathrm{j}} c_{\mathrm{j}}^{\text {bulk }} / \rho^{\mathrm{s}}\right)
$$

Eqn. 33 exhibits a linear dependence of $\Delta \rho / \rho^{\mathrm{s}}$ on concentration, consistent with the experimental results of Amatore et $\mathrm{al}^{3}$ who observed linearity of the steady-state current for the oxidation of $\mathrm{Fe}(\mathrm{CN})_{6}^{4-}$ (with a given electrode orientation) as long as $\left[\mathrm{Fe}(\mathrm{CN})_{6}^{4-}\right] \leq 0.01 \underline{\mathrm{M}}$. The PMVs for $\mathrm{Fe}(\mathrm{CN})_{6}^{4-}, \mathrm{Fe}(\mathrm{CN})_{6}^{3-}$ and $\mathrm{K}^{+}$are 96,140 and $3.5 \mathrm{~cm}^{3} /$ mole respectively; the corresponding $M_{\mathrm{j}}$ values are: $211.9,211.9$ and 39.1. With $\left[\mathrm{Fe}(\mathrm{CN})_{6}^{4-}\right]=1 \mathrm{e}-5$ moles $\mathrm{cm}^{-3}$. Thus, at a concentration of $1 \mathrm{e}-5 \underline{\mathrm{M}}$ the conditions of eqn. 32 are easily met and eqn. 33 is the operative 
expression for $\Delta \rho / \rho^{\mathrm{S}}$. One implication is that the convectively induced current component will be proportional to $\Delta \rho$ and that will be true only as long as the system is at steady-state or as long as the dimensions of the depletion layer are the same. However, it should be noted that the resultant value of $\Delta \rho / \rho^{\mathrm{S}}$ is much smaller than unity and involves taking the difference of two larger and (usually) positive terms. As noted earlier it is possible for $\beta_{\mathrm{j}} \mathrm{c}_{\mathrm{j}}$ values to be negative $^{9-}$ ${ }^{11} ; M_{\mathrm{j}} \mathrm{c}_{\mathrm{j}}$ terms are always positive.

\section{Conclusions.}

The present analysis offers some theoretical insights regarding the principle of unchanging total concentration in multispecies systems ${ }^{1}$. One of the interesting consquences of the application of that principle is that the surface concentrations of all species (the redox pair and any number of non-electroactive ions) can be definitively deduced for a given interfacial perturbation. To the best of my knowledge has been done only for a 3-species system comprising the redox pair and a counterion. The generality and simplicity of the approach is both surprising and vexing - I was concerned that the formalism I used might not be producing a unique result. However, direct explicit finite-difference simulation confirmed the result and, by implication, also confirmed the validity of the use of the Boltzmann expression (eqn. 16) to correlate $c_{j}^{x=0}$ and $c_{j}^{\text {bulk }}$ for $\mathrm{j} \geq 3$. A rather simplistic analysis was invoked to describe how the partial molar volumes $\left(\beta_{\mathrm{j}}\right)$, molecular masses $\left(M_{\mathrm{j}}\right)$, bulk concentrations $\left(c_{\mathrm{j}}^{\text {bulk }}\right)$ and the surface concentrations $\left(c_{\mathrm{j}}^{x=0}\right)$ might couple to produce a change in the density at the electrode surface. The mathematical expression for that is (see eqn. 31 and associated discussion):

$$
\frac{\Delta \rho}{\rho^{\mathrm{s}}}=\frac{1+\sum_{\mathrm{j}=1}^{\mathrm{j} \max } M_{\mathrm{j}} c_{\mathrm{j}}^{x=0} / \rho^{\mathrm{s}}}{1+\sum_{\mathrm{j}=1}^{\mathrm{j} \max } \beta_{\mathrm{j}} c_{\mathrm{j}}^{x=0}}-\frac{1+\sum_{\mathrm{j}=1}^{\mathrm{j} \max } c_{\mathrm{j}}^{\text {bulk }} / \rho^{\mathrm{s}}}{1+\sum_{\mathrm{j}=1}^{\text {jmax }} \beta_{\mathrm{j}} c_{\mathrm{j}}^{\text {bulk }}}
$$

The values of $\beta_{\mathrm{j}}, M_{\mathrm{j}}, c_{\mathrm{j}}^{\mathrm{bulk}}$ and $c_{\mathrm{j}}^{x=0}$ are likely to be such that the conditions of eqns. 32 will generally be fulfilled and the expression for the change in density simplifies to (see eqn. 33 and associated discussion): 
$\Delta \rho / \rho^{\mathrm{s}} \cong\left(\sum_{\mathrm{j}=1}^{\mathrm{j} \max } \beta_{\mathrm{j}} c_{\mathrm{j}}^{\text {bulk }}+\sum_{\mathrm{j}=1}^{\mathrm{j} \max } M_{\mathrm{j}} \mathrm{c}_{\mathrm{j}}^{x=0} / \rho^{\mathrm{s}}\right)-\left(\sum_{\mathrm{j}=1}^{\mathrm{j} \max } \beta_{\mathrm{j}} c_{\mathrm{j}}^{x=0}+\sum_{\mathrm{j}=1}^{\mathrm{j} \max } M_{\mathrm{j}} c_{\mathrm{j}}^{\text {bulk }} / \rho^{\mathrm{s}}\right)$

I say "simplistic" - because the underlying principle of the analysis is based on the premise that the diffusion coefficients of all species are identical and that is certainly not likely to be the case. That shortcoming can be alleviated by carrying out a full simulation which can take into account the size and shape of the electrode, the time duration (if the system is not at steady-state), individual $D_{\mathrm{j}}$ values and the density-gradient-induced convection near the electrode surface. The driving force for the convection will be the density gradient which will depend upon $\Delta \rho$ and the dimensions of the depletion region. Just how will the magnitude of this density-gradient induced current compare to the current attributed to natural convection?

\section{Acknowledgement.}

My thanks to Henry White, Martin Edwards, Kim McKelvey, Ernie Lewis, Hugh Isaacs and Irina Svir for helpful comments. I'd also like to thank Prof. White for his invitation for an extended visit with the White group and for his hospitality. John Miller and the Chemistry Department, Brookhaven National Laboratory are thanked for their support of my guest appointment.

\section{References.}

(1) Oldham, K. B.; Feldberg, S. W. J. Phys. Chem. B 1999, 103, 1699.

(2) Bard, A. J.; Faulkner, L. R. Electrochemical Methods: Fundamentals and Applications Second Edition; John Wiley and Sons: New York, 2001.

(3) Amatore, C.; Szunerits, S.; Thouin, L.; Warkocz, J.-S. Journal of electroanalytical chemistry 2001, 50062.

(4) Kolthoff, I. M.; Lingane, J. J. Chem. Rev. 1939, $24,1$.

(5) Drew, S. M.; Wightman, R. M.; Amatore, C. A. Journal of electroanalytical chemistry 1991, 317, 117.

(6) Gao, X.; Lee, J.; White, H. S. Analytical Chemistry 1995, 67, 1541.

(7) Bond, A. M.; Feldberg, S. W. J. Phys. Chem. B 1998, 102, 9966.

(8) Bond, A. M.; Coomber, D. C.; Feldberg, S. W.; Vu, T. Analytical Chemistry 2001, 73, 352.

(9) Zana, R.; Yeager, E. J. Phys. Chem.

1966, $70,954$.

(10) Marcus, Y. J. Chem. Soc., Faraday Trans. 1993, 89, 713.

(11) Marcus, Y.; Hefter, G.; Pang, T.-S. J. Chem. SOc. Faraday Grans. 1994, 90, 1899. 
(12) Kiselev, V. D.; Potapova, L. N.; Kashaeva, H. A.; Konovalov, A. I. Mendeleev Commun. 2012, 22, 50. 
Table 1. Values of $c_{\mathrm{j}}^{x=0} / c_{1}^{\text {buk }}$ computed for some examples of the 3-species problem.

\begin{tabular}{|c|c|c|c|c|c|c|c|}
\hline \# & $\mathrm{J}$ & $z(\mathrm{j})$ & $c_{\mathrm{j}}^{\text {bulk }} / c_{1}^{\text {bulk }}$ & $\begin{array}{l}c_{\mathrm{j}}^{x=0} / c_{1}^{\text {bulk }} \\
\alpha_{\mathrm{D}}=0.25 \\
\theta_{\mathrm{D}}=1 / 3\end{array}$ & $\begin{array}{l}c_{\mathrm{j}}^{x=0} / c_{1}^{\text {bulk }} \\
\alpha_{\mathrm{D}}=0.5 \\
\theta_{\mathrm{D}}=1.0\end{array}$ & $\begin{array}{l}c_{\mathrm{j}}^{x=0} / c_{1}^{\text {bulk }} \\
\alpha_{\mathrm{D}}=0.75 \\
\theta_{\mathrm{D}}=3\end{array}$ & $\begin{aligned} c_{\mathrm{j}}^{x=0} / c_{1}^{\text {bulk }} \\
\alpha_{\mathrm{D}}=0.999999 \\
\theta_{\mathrm{D}}=1 \mathrm{e} 6\end{aligned}$ \\
\hline \multirow{4}{*}{$1 \mathrm{~A}$} & 1 & 1 & 1 & $6 / 7$ & $2 / 3$ & $2 / 5$ & $2 \mathrm{e}-6$ \\
\hline & 2 & 0 & 0 & $2 / 7$ & $2 / 3$ & $6 / 5$ & 2 \\
\hline & 3 & -1 & 1 & $6 / 7$ & $2 / 3$ & $2 / 5$ & $2 \mathrm{e}-6$ \\
\hline & \multicolumn{3}{|r|}{$\gamma=$} & -0.1542 & -0.4054 & -0.9163 & 13.12 \\
\hline\|\|\|\| & $\|$ & $|\||||||$ & |||||||||||||||||| & ||||||||||||||||||||||||||||||||| & |||||||||||||||||||||||||||||||||| & |||||||||||||||||||||||| & $|\||||||||||||||||||||||||||||$ \\
\hline \multirow{4}{*}{ 1B } & 1 & 1 & 1 & $2 / 3$ & $2 / 5$ & $2 / 11$ & $6.66667 \mathrm{e}-7$ \\
\hline & 2 & 2 & 0 & $2 / 9$ & $2 / 5$ & $6 / 11$ & $2 / 3$ \\
\hline & 3 & -1 & 1 & $1 / 9$ & $6 / 5$ & $14 / 11$ & $4 / 3$ \\
\hline & & & $\gamma=$ & -0.1054 & 0.1823 & 0.2412 & 0.2877 \\
\hline
\end{tabular}


Table 2. V values of $c_{\mathrm{j}}^{x=0} / c_{1}^{\text {buk }}$ computed for some examples of the 5-species and 4-species systems. Data in parentheses were computed using explicit finite difference simulation. ${ }^{7}$

\begin{tabular}{|c|c|c|c|c|c|c|c|}
\hline \# & $\mathrm{J}$ & $z(\mathrm{j})$ & $c_{\mathrm{j}}^{\text {bulk }} / c_{1}^{\text {bulk }}$ & $\begin{array}{l}c_{\mathrm{j}}^{x=0} / c_{1}^{\text {bulk }} \\
\alpha_{\mathrm{D}}=0.25 \\
\theta_{\mathrm{D}}=1 / 3\end{array}$ & $\begin{array}{l}c_{\mathrm{j}}^{x=0} / c_{1}^{\text {bulk }} \\
\alpha_{\mathrm{D}}=0.5 \\
\theta_{\mathrm{D}}=1.0\end{array}$ & $\begin{array}{l}c_{\mathrm{j}}^{x=0} / c_{1}^{\text {bulk }} \\
\alpha_{\mathrm{D}}=0.75 \\
\theta_{\mathrm{D}}=3\end{array}$ & $\begin{aligned} c_{\mathrm{j}}^{x=0} / c_{1}^{\text {bulk }} \\
\alpha_{\mathrm{D}}=0.999999 \\
\theta_{\mathrm{D}}=1 \mathrm{e} 6\end{aligned}$ \\
\hline \multirow{6}{*}{$2 \mathrm{~A}$} & 1 & 1 & 1 & 0.7500820 & $\begin{array}{l}0.5000937 \\
(0.50252)\end{array}$ & 0.2500586 & $\begin{array}{l}1.00025 \mathrm{e}-6 \\
(1.6135 \mathrm{e}-6)\end{array}$ \\
\hline & 2 & 0 & 0 & 0.2500273 & $\begin{array}{l}0.5000937 \\
(0.497680)\end{array}$ & 0.7501757 & $\begin{array}{c}1.00025 \\
(1.0003139)\end{array}$ \\
\hline & 3 & -1 & 1 & 0.9998751 & $\begin{array}{c}0.9997502 \\
(0.99975)\end{array}$ & 0.9996253 & $\begin{array}{c}0.9995 \\
(0.9995)\end{array}$ \\
\hline & 4 & 1 & 1000 & $1.000125 \mathrm{e}+3$ & $\begin{array}{l}1.00025 \mathrm{e}+3 \\
(1.000249)\end{array}$ & $1.0003749 \mathrm{e}+3$ & $\begin{array}{c}1.0005 \mathrm{e} 3 \\
(1.0005 \mathrm{e} 3)\end{array}$ \\
\hline & 5 & -1 & 1000 & $9.998751 \mathrm{e}+2$ & $\begin{array}{l}0.99975 \mathrm{e}+3 \\
(0.99975 \mathrm{e} 3)\end{array}$ & $9.9962529+2$ & $\begin{array}{c}0.9995 \mathrm{e}+3 \\
(0.9995 \mathrm{e}+3)\end{array}$ \\
\hline & & & $\gamma=$ & $-1.249 \mathrm{e}-4$ & $-2.498 \mathrm{e}-4$ & $-3.748 \mathrm{e}-4$ & $-4.998 \mathrm{e}-4$ \\
\hline\|\|\|\| & $\|$ & \|\|\|\|$\|$ & $|\|||||||||||||||| \mid$ & $|\||||||||||||||||||||||||||||||||$ & |||||||||||||||||| & $|\|||||||||||||||||||||| \mid$ & $|\||||||||||||||||||||||||||||||||$ \\
\hline \multirow{5}{*}{$2 \mathrm{~B}$} & 1 & 1 & 1 & 0.7500819 & 0.5000937 & 0.2498714 & $9.992519 \mathrm{e}-7$ \\
\hline & 2 & 2 & 0 & 0.2500273 & 0.5000937 & 0.7496141 & $9.99251 \mathrm{e}-1$ \\
\hline & 3 & -1 & 1001 & $1.000875 \mathrm{e}+3$ & 1.000750 & $1.001374 \mathrm{e} 3$ & $1.001500+3$ \\
\hline & 4 & 1 & 1000 & $1.0001249 \mathrm{e}+3$ & 1.00250 & $0.9996257+03$ & $0.9995011+3$ \\
\hline & & & $\gamma=$ & $-1.249 \mathrm{e}-4$ & $-2.498 \mathrm{e}-4$ & $-3.744 \mathrm{e}-4$ & $-4.997 \mathrm{e}-4$ \\
\hline
\end{tabular}

